

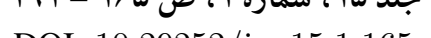

DOI: $10.29252 /$ jss.15.1.165

مقاله يُوهشى

خانواده توزيعهاى وايبل تعميميافته تركيبى با توزيع دوجملهاى منفى بريده در صفر

$$
\begin{aligned}
& \text { بهرام طارمى'، محسن آوجى'، ناهيد سنجرى فارسى يورّ } \\
& \text { 'بخش آمار، دانشكده علوم، دانشعاه شيراز } \\
& \text { كَروه رياضى كاربردى، دانشكده علوم رياضى، دانشكاه تبريز } \\
& \text { كروه آمار، دانشكده علوم رياضى، دانشكاه الزهرا }
\end{aligned}
$$

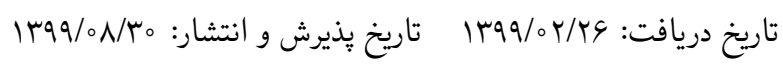

جكيده: در اين مقاله با استفاده از خانواده توزيعهاى مارشال-اولكين-ناداراجه تعميم يافته وايبل،

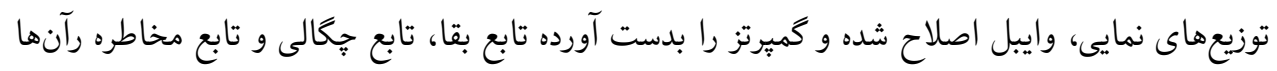



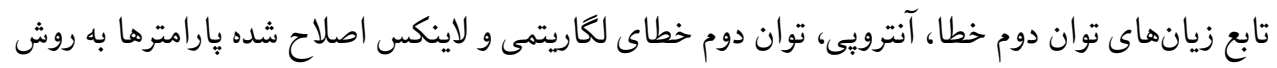

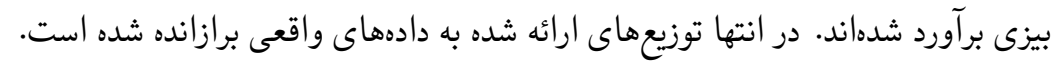

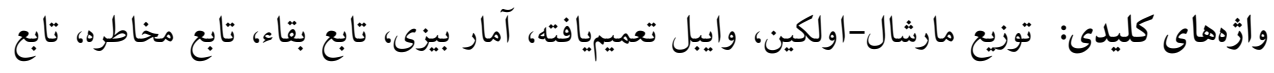

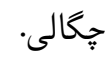

وازههاى كليدى: توزيع مارشال-اولكين، وايبل تعميميافته، آمار بيزى، تابع بقاء، تابع مخاطره.




مارشال اولكين دست يافتند. آنها با استفاده از (x)

$$
\bar{G}(x ; a)=\frac{a \bar{F}(x)}{F(x)+a \bar{F}(x)}, x \in \mathbb{R}, a>\circ,
$$

معرفى كردند. يك خاصيت جالب اين توزيع اين است كه اخر متغير تصادفى N داراى توزيع هندسى با قارامتر $\alpha$ و تابع جرم احتمال

$$
\begin{aligned}
& \mathrm{P}(N=n)=\alpha(1-\alpha)^{n-1}, n=1, \mathrm{r}, \ldots, \quad \alpha \in(\circ, 1) \\
& \mathrm{T}(N=n)=\alpha^{-1}\left(1-\alpha^{-1}\right)^{n-1}, n=1, r, \ldots, \quad \alpha>1
\end{aligned}
$$

باشد، آنكاه $)$

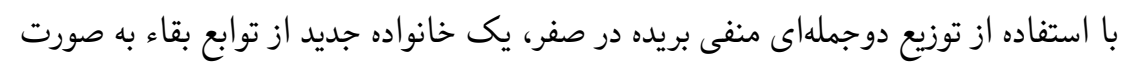

$$
\bar{G}(x ; a, b)=\frac{a^{b}}{1-a^{b}}\left[(F(x)+a \bar{F}(x))^{-b}-1\right], x \in \mathbb{R}, a, b>。
$$

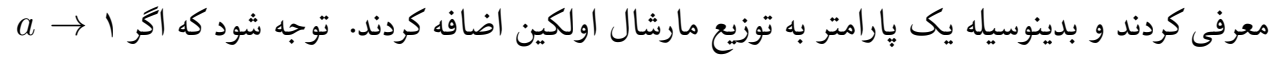
آنكاه

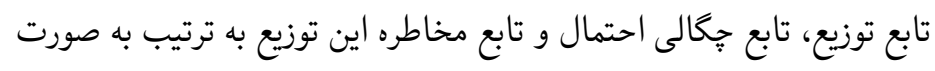

$$
\begin{aligned}
G(x ; a, b) & =\frac{[1-\bar{F}(x) \bar{a}]^{b}-a^{b}}{[1-\bar{F}(x) \bar{a}]^{b}\left(1-a^{b}\right)}, \\
g(x ; a, b) & =\frac{\bar{a} b a^{b} f(x)}{[1-\bar{F}(x) \bar{a}]^{b+1}\left(1-a^{b}\right)}, \\
r_{G}(x ; a, b) & =\frac{\bar{a} b \bar{F}(x) r_{F}(x)}{[1-\bar{F}(x) \bar{a}]\left[1-(F(x)+a \bar{F}(x))^{b}\right]},
\end{aligned}
$$


هستند، كه در آن a - 1 شكل (x) هن همجنين

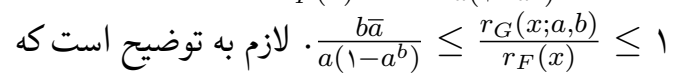

$$
\begin{aligned}
& \lim _{x \rightarrow-\infty} r_{G}(x ; a, b)=\frac{b \bar{a}}{a\left(1-a^{b}\right)} \lim _{x \rightarrow-\infty} r_{F}(x), \\
& \lim _{x \rightarrow \infty} r_{G}(x ; a, b)=\lim _{x \rightarrow \infty} r_{F}(x) .
\end{aligned}
$$

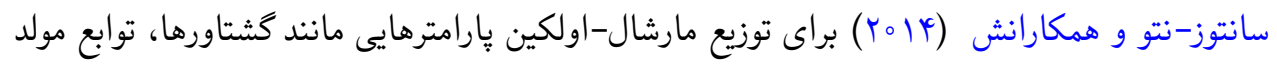

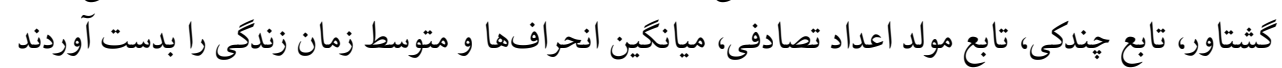



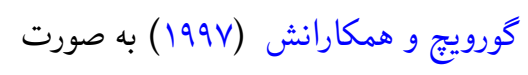

$$
F(x ; \alpha, \boldsymbol{\xi})=1-\exp [-\alpha H(x ; \boldsymbol{\xi})], x \in D \subseteq \mathbb{R}^{+}, \alpha>\circ,
$$

معرفى شد، در مدلهاى زتدگى و بقاء مورد استفاده قرار گرفته است. رابطه (Y) براى حالت خاص

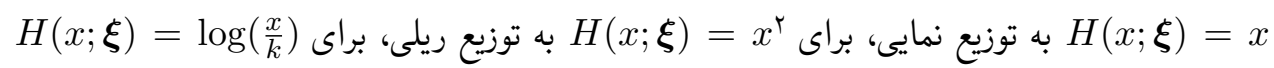
به توزيع يارتو و براى [1 - -

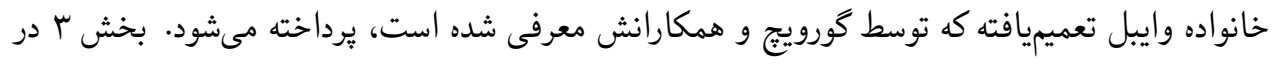

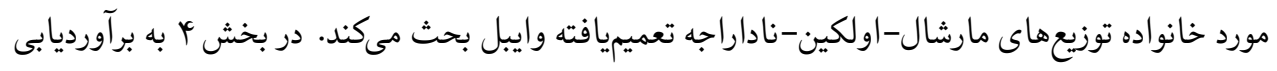

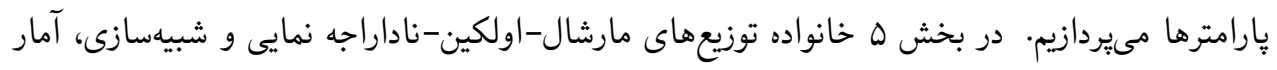

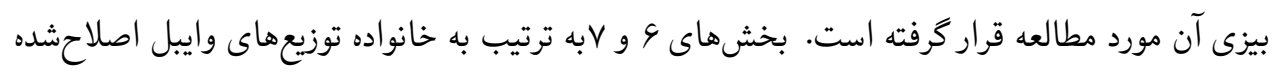


نتيجهيرى ارائه شده است. 


\section{خ ب انواده وايبل تعميميافته}

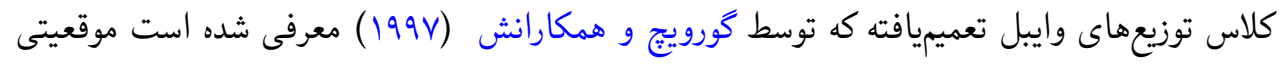

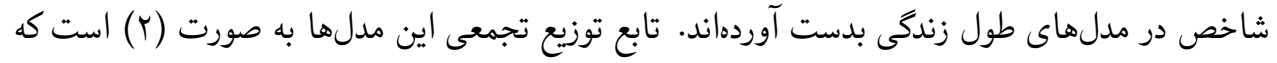

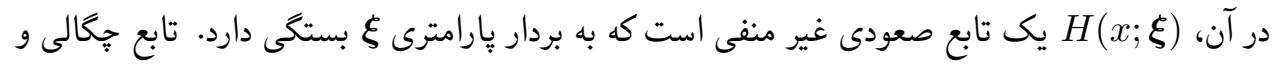



$$
\begin{aligned}
& f(x ; \alpha, \boldsymbol{\xi})=\alpha h(x ; \boldsymbol{\xi}) \exp [-\alpha H(x ; \boldsymbol{\xi})], \\
& \bar{F}(x ; \alpha, \boldsymbol{\xi})=\exp [-\alpha H(x ; \boldsymbol{\xi})]
\end{aligned}
$$

هستند، كه در آن (H)

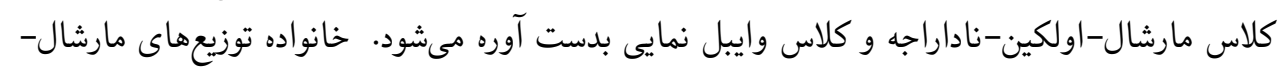

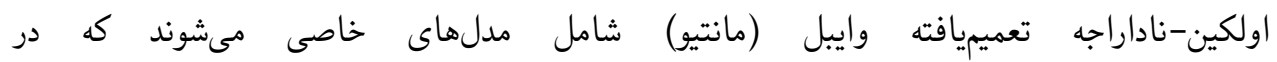

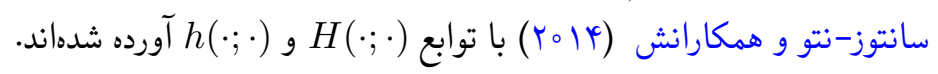

خ خانواده توزيعهاى مانتيو

$$
\text { تابع توزيع اين خانواده به صورت }
$$

$$
G(x ; a, b, \alpha, \boldsymbol{\xi})=\frac{[1-\bar{a} \exp [-\alpha H(x ; \boldsymbol{\xi})]]^{b}-a^{b}}{\left(1-a^{b}\right)[1-\bar{a} \exp [-\alpha H(x ; \boldsymbol{\xi})]]^{3}},
$$




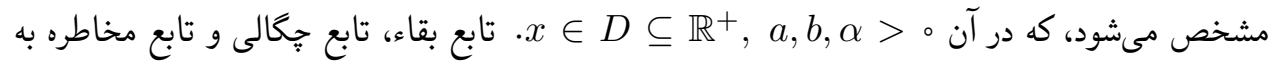

$$
\begin{aligned}
& \bar{G}(x ; a, b, \alpha, \boldsymbol{\xi})=\frac{a^{b}-a^{b}[1-\bar{a} \exp [-\alpha H(x ; \boldsymbol{\xi})]]^{b}}{\left(1-a^{b}\right)[1-\bar{a} \exp [-\alpha H(x ; \boldsymbol{\xi})]]^{b}}, \\
& g(x ; a, b, \alpha, \boldsymbol{\xi})=\frac{a^{b} b \bar{a} \alpha h(x ; \boldsymbol{\xi}) \exp [-\alpha H(x ; \boldsymbol{\xi})]}{\left(1-a^{b}\right)[1-\bar{a} \exp [-\alpha H(x ; \boldsymbol{\xi})]]^{b+1}} \\
& r_{G}(x ; a, b, \alpha, \boldsymbol{\xi})=\frac{b \bar{a} \alpha h(x ; \boldsymbol{\xi}) \exp [-\alpha H(x ; \boldsymbol{\xi})]}{(1-\bar{a} \exp [-\alpha H(x ; \boldsymbol{\xi})])\left(1-[1-\bar{a} \exp [-\alpha H(x ; \boldsymbol{\xi})]]^{b}\right)}, \\
& \text { تعيين مىشوند. براى عدد حقيقى و مثبت r و | > |z|، بسط دوجملهاى تعميميافته به صورت } \\
& (1-z)^{-r}=\sum_{k=。}^{\infty} \frac{(r)_{k}}{k !} z^{k}
\end{aligned}
$$$$
\text { ترتيب به صورت }
$$

است، كه در آن (r)

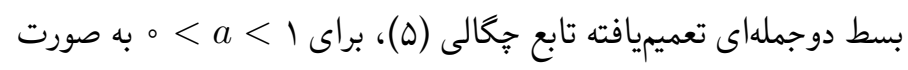

$$
g(x ; a, b, \alpha, \boldsymbol{\xi})=\sum_{j=\circ}^{\infty} \eta_{j} f(x,(j+1) \alpha, \boldsymbol{\xi})
$$

است، كه در آن ان a> $a$ داريم

$$
g(x ; a, b, \alpha, \boldsymbol{\xi})=\frac{a^{b} b \bar{a} \alpha h(x ; \boldsymbol{\xi}) \exp [-\alpha H(x ; \boldsymbol{\xi})]}{\left(1-a^{b}\right) a^{b+1}\left(1-\left(1-\frac{1}{a}\right)\{1-\exp [-\alpha H(x ; \boldsymbol{\xi})]\}\right)^{b+1}}
$$


در اين حالت مىتوان نشان داد > > (I) (1) معادله (9) داريم

$$
g(x ; a, b, \alpha, \boldsymbol{\xi})=\sum_{j=\circ}^{\infty} \nu_{j} f(x,(j+1) \alpha, \boldsymbol{\xi})
$$

كه در آن (1) (1) (1)

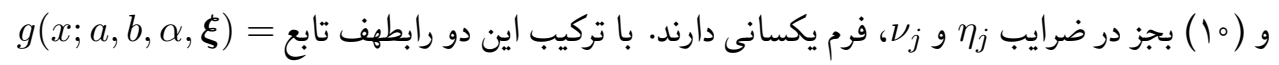

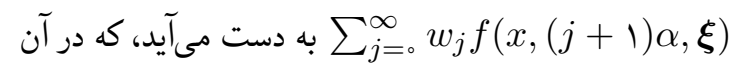

$$
w_{j}= \begin{cases}\eta_{j}, & 0<a<1 \\ \nu_{j}, & a>1\end{cases}
$$

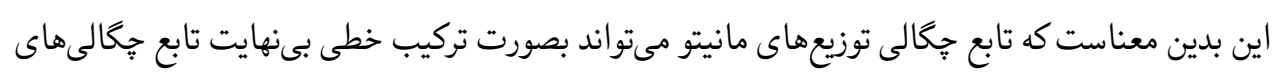

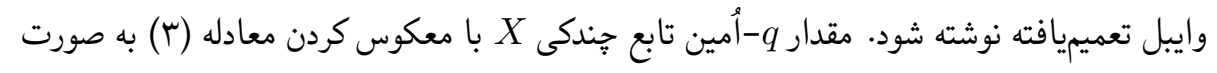

$$
Q(u)=H^{-1}\left(\frac{1}{\alpha} \log \frac{\bar{a}\left[1-u\left(1-a^{b}\right)\right]}{\left[1-u\left(1-a^{b}\right)\right]^{\frac{1}{b}}-a}\right)
$$

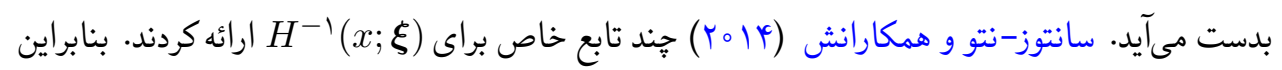
براى توليد عدد تصادفى از توزيع X X، الكوريتم زير قابل استفاده است.

الكوريتم ا. توليد اعداد تصادفى براى توزيعهاى مانيتويِ

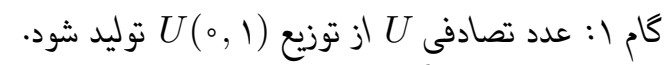
كام ץ: مقدار

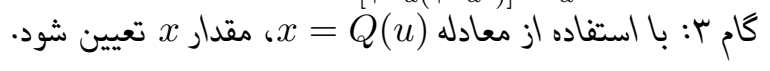


بهرام طارمى، محسن آوجى، ناهيد سنجرى فارسى يور

\section{ب برآورد بارامترهاى توزيعهاى مانتيو}

اخر

$$
L(a, b, \alpha, \boldsymbol{\xi})=\frac{a^{n b} b^{n} \bar{a}^{n} \alpha^{n} \prod_{i=1}^{n} h\left(x_{i} ; \boldsymbol{\xi}\right) \exp \left[-\alpha \sum_{i=1}^{n} H\left(x_{i} ; \boldsymbol{\xi}\right)\right]}{\left(1-a^{b}\right)^{n} \prod_{i=1}^{n}\left[1-\bar{a} \exp \left[-\alpha H\left(x_{i} ; \boldsymbol{\xi}\right)\right]\right]^{b+1}}
$$

$$
\text { و لعاريتم آن نيز بصورت }
$$

$$
\begin{aligned}
& \ell(a, b, \alpha, \boldsymbol{\xi})=n b \log a+n \log b+n \log \bar{a}+n \log \alpha+\sum_{i=1}^{n} \log h\left(x_{i} ; \boldsymbol{\xi}\right) \\
& -\alpha \sum_{i=1}^{n} H\left(x_{i} ; \boldsymbol{\xi}\right)-n \log \left(1-a^{b}\right)-\sum_{i=1}^{n}(b+1) \log \left[1-\bar{a} \exp \left[-\alpha H\left(x_{i} ; \boldsymbol{\xi}\right)\right]\right]
\end{aligned}
$$

هستند. مشتقهاى جزئى لكاريتم درستنمايى نسبت به پارامترهاى توزيع عبارتند از

$$
\begin{aligned}
\frac{\partial \ell}{\partial a} & =\frac{n b}{a}-\frac{n}{\bar{a}}+\frac{n b a^{b-1}}{1-a}-\sum_{i=1}^{n}(b+1) \frac{\exp \left[-\alpha H\left(x_{i} ; \boldsymbol{\xi}\right)\right]}{1-\bar{a} \exp \left[-\alpha H\left(x_{i} ; \boldsymbol{\xi}\right)\right]} \\
\frac{\partial \ell}{\partial b} & =n \log a+\frac{n}{b}+\frac{n a^{b} \log a}{1-a^{b}}-\sum_{i=1}^{n} \log \left[1-\bar{a} \exp \left[-\alpha H\left(x_{i} ; \boldsymbol{\xi}\right)\right]\right] \\
\frac{\partial \ell}{\partial \alpha} & =\frac{n}{\alpha}-\sum_{i=1}^{n} H\left(x_{i} ; \boldsymbol{\xi}\right)-\sum_{i=1}^{n}(b+1) \frac{\bar{a} H\left(x_{i}, \boldsymbol{\xi}\right) \exp \left[-\alpha H\left(x_{i} ; \boldsymbol{\xi}\right)\right]}{1-\bar{a} \exp \left[-\alpha H\left(x_{i} ; \boldsymbol{\xi}\right)\right]} \\
\frac{\partial \ell}{\partial \xi_{k}} & =\sum_{i=1}^{n} \frac{\frac{\partial h\left(x_{i} ; \boldsymbol{\xi}\right)}{\partial \xi_{k}}}{h\left(x_{i} ; \boldsymbol{\xi}\right)}-\alpha \sum_{i=1}^{n} \frac{\partial H\left(x_{i} ; \boldsymbol{\xi}\right)}{\partial \xi_{k}}-\sum_{i=1}^{n}(b+1) \frac{\bar{a} \alpha \frac{\partial H\left(x_{i} ; \boldsymbol{\xi}\right)}{\partial \xi_{k}} \exp \left[-\alpha H\left(x_{i} ; \boldsymbol{\xi}\right)\right]}{\left[1-\bar{a} \exp \left[-\alpha H\left(x_{i} ; \boldsymbol{\xi}\right)\right]\right]}
\end{aligned}
$$


بر اساس مشتقهاى مرتبه دوم، ماتريس اطلاعات افراز شده براى خانواده توزيعهاى مانتيو به صورت

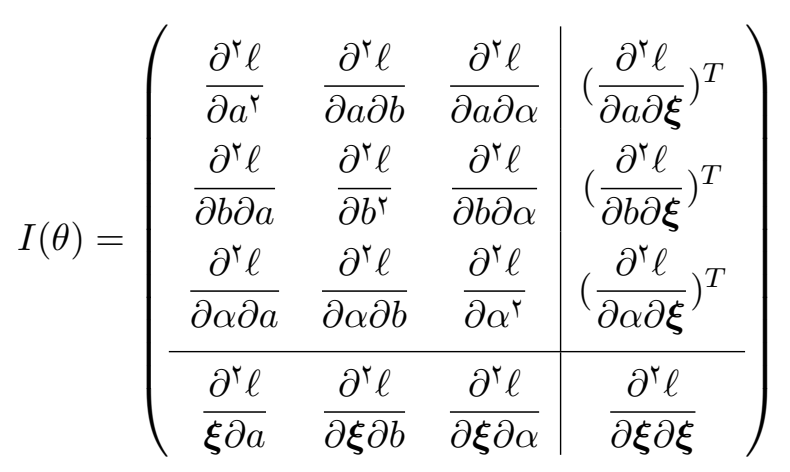

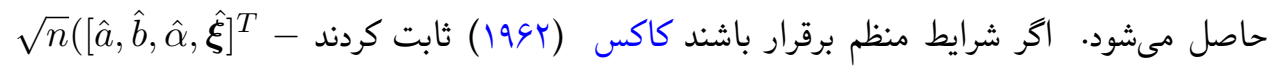

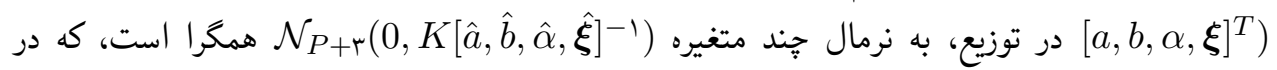

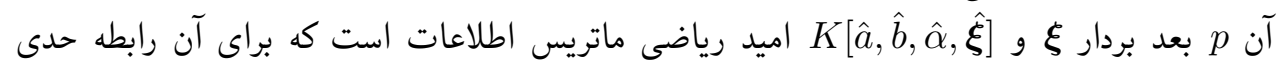

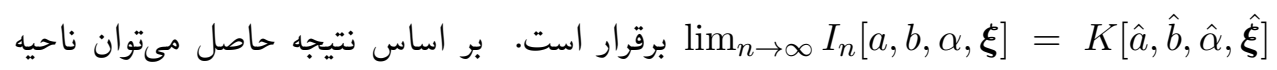

$$
\text { اطمينان را براى پارامترهاى توزيعهاى مانتيو بدست آورد. }
$$

\section{هانو اده توزيعهاى مارشال - اولكين - ناداراجه نمايى (مانن)}

در اين خانواده از توزيعها،



$$
\begin{aligned}
& G(x ; a, b, \alpha)=\frac{[1-\bar{a} \exp [-\alpha x]]^{b}-a^{b}}{\left(1-a^{b}\right)[1-\bar{a} \exp [-\alpha x]]^{b}}, \\
& \bar{G}(x ; a, b, \alpha)=\frac{a^{b}-a^{b}[1-\bar{a} \exp [-\alpha x]]^{b}}{\left(1-a^{b}\right)[1-\bar{a} \exp [-\alpha x]]^{b}}, \\
& g(x ; a, b, \alpha)=\frac{a^{b} b \bar{a} \alpha \exp [-\alpha x]}{\left(1-a^{b}\right)[1-\bar{a} \exp [-\alpha x]]^{b+1}}, \\
& r_{G}(x ; a, b, \alpha)=\frac{b \bar{a} \alpha \exp [-\alpha x]}{[1-\bar{a} \exp [-\alpha x]]\left\{1-[1-\bar{a} \exp [-\alpha x]]^{b}\right\}} .
\end{aligned}
$$


$a=\frac{1}{r}, b=$ در شكل ا، نمودارهاى تابع توزيع، تابع بقا، تابع جالى و تابع مخاطره براى دو حالت



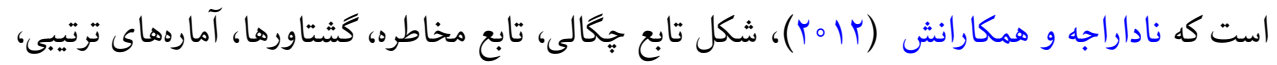

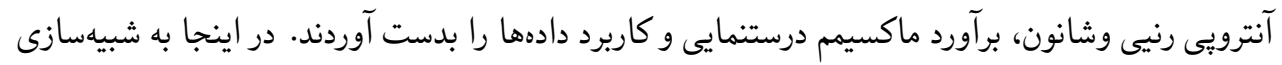

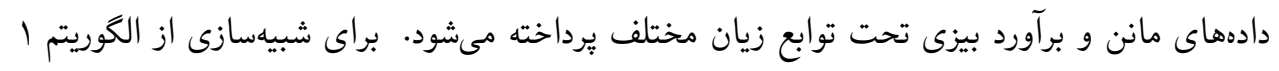

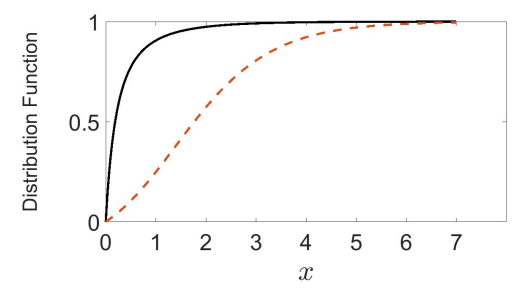

(ب)

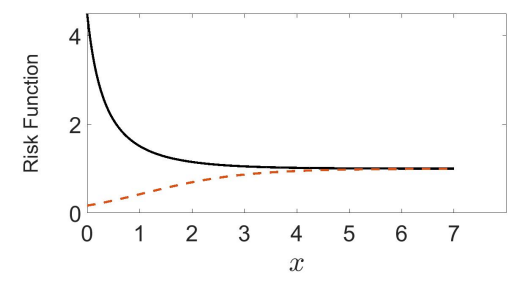

(2)

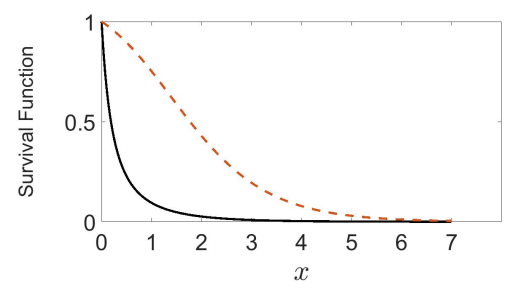

(الف)

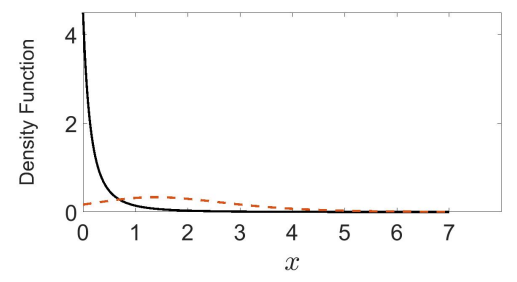

(ج)

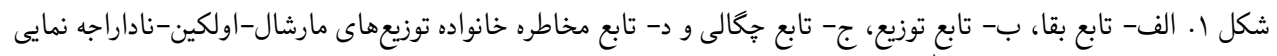

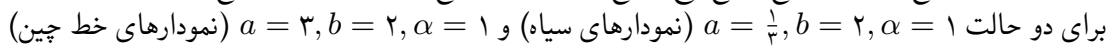

$$
\begin{aligned}
& \text { استفاده مىشود. در اينجا }=x \text { إست } H^{-1} \text { است. بنابراين } \\
& X=\frac{1}{\alpha} \log \frac{\bar{a}\left[1-U\left(1-a^{b}\right)\right]}{\left[1-U\left(1-a^{b}\right)\right]^{\frac{1}{b}}-a}
\end{aligned}
$$

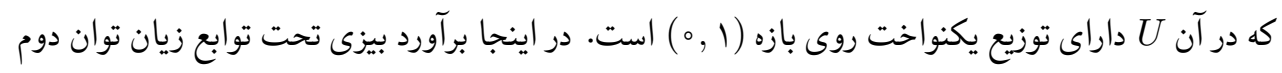

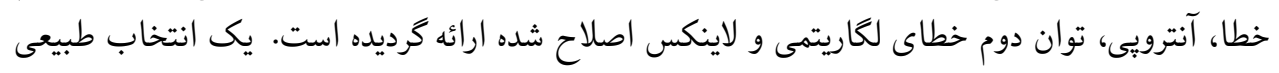

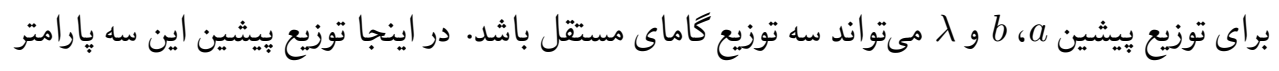
را به ترتيب (Gamma( 

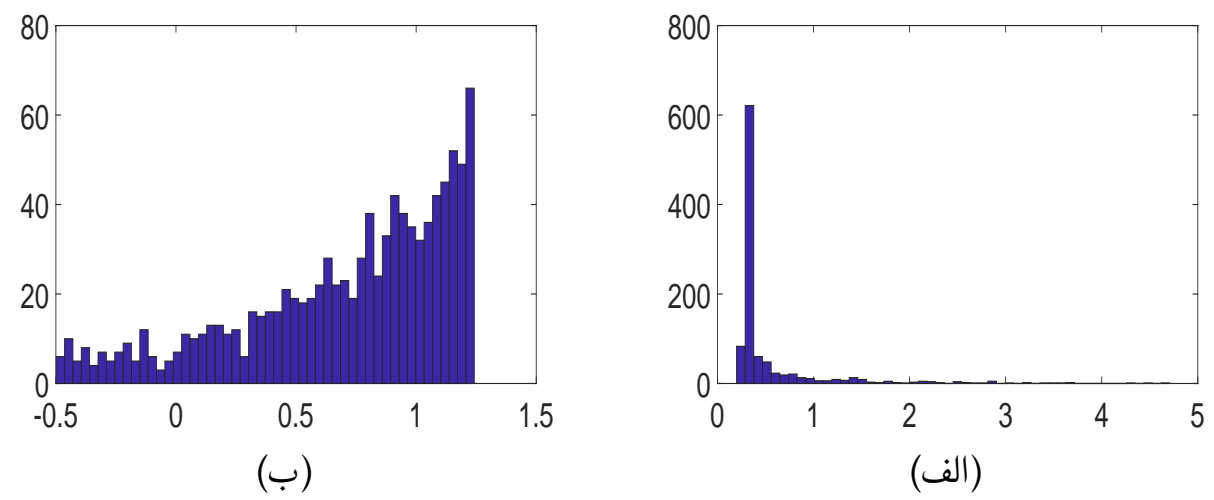



جگالى احتمال توام بيشين بارامترهاى a، b و

$f_{\alpha, \beta, \theta, \gamma, \eta, \varepsilon}(a, b, \lambda) \propto a^{\alpha-1} b^{\theta-1} \lambda^{\eta-1} e^{-a \beta-b \gamma-\lambda \varepsilon}, \quad \alpha, \beta, \theta, \gamma, \eta, \varepsilon, a, b, \lambda \geq 。$

است. بنابراين تابع جگالى پِين a، b و

$$
\begin{aligned}
f(a, b, \lambda \mid x) & =k a^{\alpha-1} b^{\theta-1} \lambda^{\eta-1} e^{-a \beta-b \gamma-\lambda \varepsilon} \frac{a^{n b} b^{n} \bar{a}^{n} \lambda^{n} e^{-\lambda \sum_{i=1}^{n} x_{i}}}{\left(1-a^{b}\right)^{n}} \\
& \times \prod_{i=1}^{n}\left[1-e^{-\lambda x_{i}} \bar{a}\right]^{-(b+1)}
\end{aligned}
$$

است، كه در آن

$$
\begin{aligned}
k^{-1} & =\int_{a} \int_{b} \int_{\lambda} a^{\alpha-1} b^{\theta-1} \lambda^{\eta-1} e^{-a \beta-b \gamma-\lambda \varepsilon} \frac{a^{n b} b^{n} \bar{a}^{n} \lambda^{n} e^{-\lambda \sum_{i=1}^{n} x_{i}}}{\left(1-a^{b}\right)^{n}} \\
& \times \prod_{i=1}^{n}\left[1-e^{-\lambda x_{i}} \bar{a}\right]^{-(b+1)} d \lambda d b d a .
\end{aligned}
$$

تابع ¥گالى يسين داراى يك انتكرال در مخرج كسر است كه به راحتى قابل حل نيست. بنابراين




استفاده مىشود. ليندلى (1910) نشان داد نسبت دو انتخرال

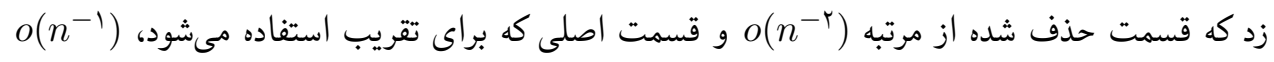

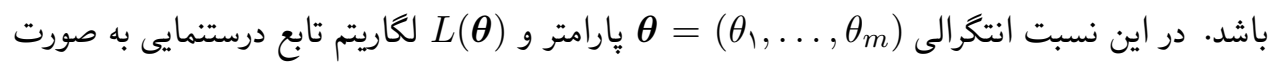

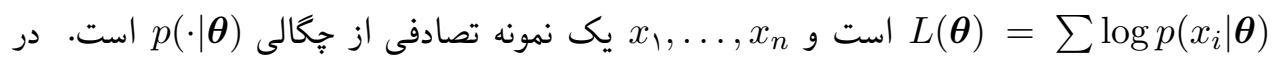
كاربرد بايد

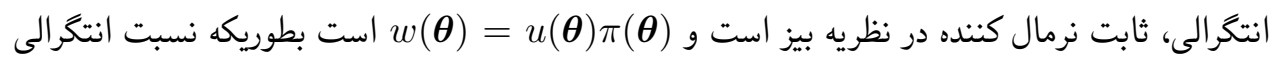
برابر

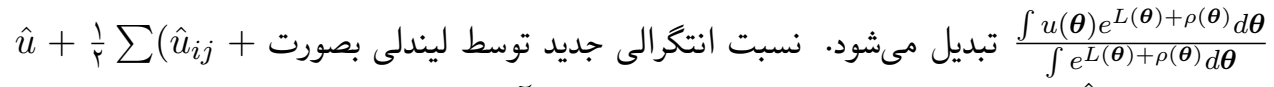

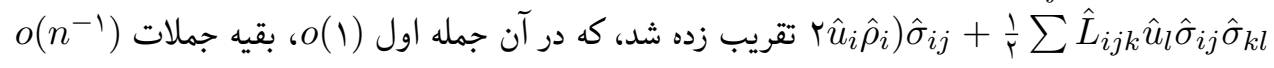
هستند و

$$
\begin{array}{ll}
\hat{u}=u(\hat{\boldsymbol{\theta}}) & \hat{g}_{i}=g_{i}(\hat{\boldsymbol{\theta}})=\left.\frac{\partial g}{\partial \theta_{i}}\right|_{\theta=\hat{\theta}}, \\
\hat{g}_{i j}=g_{i j}(\hat{\boldsymbol{\theta}})=\left.\frac{\partial^{r} g}{\partial \theta_{i} \partial \theta_{j}}\right|_{\theta=\hat{\theta}} & \hat{g}_{i j k}=g_{i j k}(\hat{\boldsymbol{\theta}})=\left.\frac{\partial^{r} g}{\partial \theta_{i} \partial \theta_{j} \partial \theta_{k}}\right|_{\theta=\hat{\theta}},
\end{array}
$$

كه در آن 年 $\theta_{r}=\lambda, \theta_{r}=b ، \theta_{1}=a ، m=r$

$$
I(x)=E(u(a, b, \lambda) \mid \mathbf{X}=\mathbf{x})=\frac{\int u(a, b, \lambda) e^{L(a, b, \lambda)+\rho(a, b, \lambda)} d a d b d \lambda}{\int e^{L(a, b, \lambda)+\rho(a, b, \lambda)} d a d b d \lambda}
$$

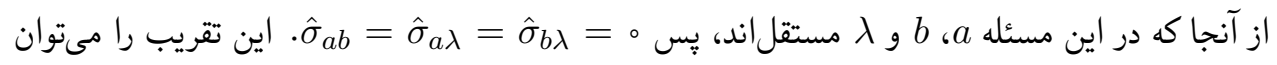
به صورت 


$$
\begin{aligned}
I(\mathbf{x})= & u(\hat{a}, \hat{b}, \hat{\lambda})+\frac{1}{r}\left[\left(\hat{u}_{a a}+r \hat{u}_{a} \hat{\rho}_{a}\right) \hat{\sigma}_{a a}+\left(\hat{u}_{b b}+r \hat{u}_{b} \hat{\rho}_{b}\right) \hat{\sigma}_{b b}\left(\hat{u}_{\lambda \lambda}+r \hat{u}_{\lambda} \hat{\rho}_{\lambda}\right) \hat{\sigma}_{\lambda \lambda}\right] \\
& +\frac{1}{r}\left[\hat{u}_{a}\left(\hat{L}_{a a a} \hat{\sigma}_{a a} \hat{\sigma}_{a a}+\hat{L}_{b b a} \hat{\sigma}_{b b} \hat{\sigma}_{a a}+\hat{L}_{\lambda \lambda a} \hat{\sigma}_{\lambda \lambda} \hat{\sigma}_{a a}\right) .\right. \\
& +\hat{u}_{b}\left(\hat{L}_{a a b} \hat{\sigma}_{a a} \hat{\sigma}_{b b}+\hat{L}_{b b b} \hat{\sigma}_{b b} \hat{\sigma}_{b b}+\hat{L}_{\lambda \lambda b} \hat{\sigma}_{\lambda \lambda} \hat{\sigma}_{b b}\right) \\
& \left.+\hat{u}_{\lambda}\left(\hat{L}_{a a \lambda} \hat{\sigma}_{a a} \hat{\sigma}_{\lambda \lambda}+\hat{L}_{b b \lambda} \hat{\sigma}_{b b} \hat{\sigma}_{\lambda \lambda}+\hat{L}_{\lambda \lambda \lambda} \hat{\sigma}_{\lambda \lambda} \hat{\sigma}_{\lambda \lambda}\right)\right]
\end{aligned}
$$

برآورد بيزى $\theta$ تحت تابع زيان توان دوم خطا بان


خطاى لكاريتمى زئم انتروبى

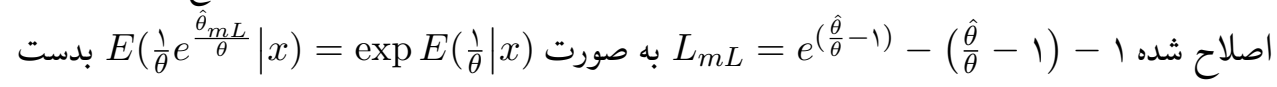

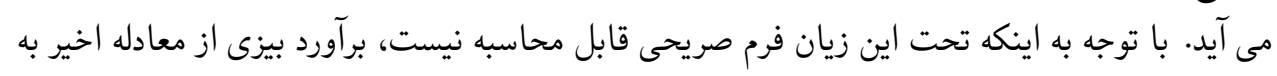

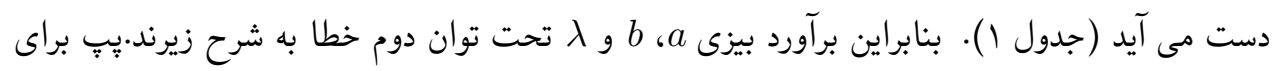
$u(a, b, \lambda)=a$

$$
\begin{aligned}
& E(a \mid x)=\hat{a}+\hat{\rho}_{a} \hat{\sigma}_{a a}+\frac{1}{r}\left[\hat{\sigma}_{a a}\left(\hat{L}_{a a a} \hat{\sigma}_{a a}+\hat{L}_{b b a} \hat{\sigma}_{b b}+\hat{L}_{\lambda \lambda a} \hat{\sigma}_{\lambda \lambda}\right)\right], \\
& E(b \mid x)=\hat{b}+\hat{\rho}_{b} \hat{\sigma}_{b b}+\frac{1}{r}\left[\hat{\sigma}_{b b}\left(\hat{L}_{b b b} \hat{\sigma}_{b b}+\hat{L}_{a a b} \hat{\sigma}_{a a}+\hat{L}_{\lambda \lambda b} \hat{\sigma}_{\lambda \lambda}\right)\right], \\
& E(\lambda \mid x)=\hat{\lambda}+\hat{\rho}_{\lambda} \hat{\sigma}_{\lambda \lambda}+\frac{1}{r}\left[\hat{\sigma}_{\lambda \lambda}\left(\hat{L}_{\lambda \lambda \lambda} \hat{\sigma}_{\lambda \lambda}+\hat{L}_{a a \lambda} \hat{\sigma}_{a a}+\hat{L}_{b b \lambda} \hat{\sigma}_{b b}\right)\right] .
\end{aligned}
$$

به همين ترتيب برآورد بيزى a، b و $\lambda$ تحت تابع زيانهاى آنترويى و توان دوم خطاى لكاريتمى در جدول ا ارائه شداند. با توجه به a $a \beta-b \gamma-\lambda \varepsilon$ $\rho_{a}=\frac{\partial \rho}{\partial a}=\frac{\alpha-1}{a}-\beta, \quad \rho_{b}=\frac{\partial \rho}{\partial b}=\frac{\theta-1}{b}-\gamma, \quad \rho_{\lambda}=\frac{\partial \rho}{\partial \lambda}=\frac{\eta-1}{\lambda}-\varepsilon$. 
جدول اـ برآورد بيزى يارامترها تحت تابع زيانهاى مختلف

\begin{tabular}{|c|c|c|}
\hline 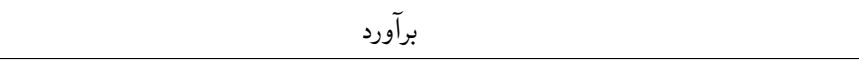 & 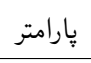 &  \\
\hline$\hat{a}+\hat{\rho}_{a} \hat{\sigma}_{a a}+\frac{1}{\gamma}\left[\hat{\sigma}_{a a}\left(\hat{L}_{a a a} \hat{\sigma}_{a a}+\hat{L}_{b b a} \hat{\sigma}_{b b}+\hat{L}_{\lambda \lambda a} \hat{\sigma}_{\lambda \lambda}\right)\right]$ & $a$ & \\
\hline$\hat{b}+\hat{\rho}_{b} \hat{\sigma}_{b b}+\frac{1}{\gamma}\left[\hat{\sigma}_{b b}\left(\hat{L}_{b b b} \hat{\sigma}_{b b}+\hat{L}_{a a b} \hat{\sigma}_{a a}+\hat{L}_{\lambda \lambda b} \hat{\sigma}_{\lambda \lambda}\right)\right]$ & $b$ & 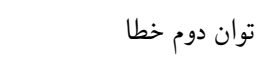 \\
\hline$\hat{\lambda}+\hat{\rho}_{\lambda} \hat{\sigma}_{\lambda \lambda}+\frac{1}{r}\left[\hat{\sigma}_{\lambda \lambda}\left(\hat{L}_{\lambda \lambda \lambda} \hat{\sigma}_{\lambda \lambda}+\hat{L}_{a a \lambda} \hat{\sigma}_{a a}+\hat{L}_{b b \lambda} \hat{\sigma}_{b b}\right)\right]$ & $\lambda$ & \\
\hline$\hat{a}\left[1+\left(\frac{1}{\hat{a}^{r}}-\frac{1}{\hat{a}} \hat{\rho}_{a}\right) \hat{\sigma}_{a a}-\frac{1}{r \hat{a}} \hat{\sigma}_{a a}\left(\hat{L}_{a a a} \hat{\sigma}_{a a}+\hat{L}_{b b a} \hat{\sigma}_{b b}+\hat{L}_{\lambda \lambda a} \hat{\sigma}_{\lambda \lambda}\right)\right]^{-1}$ & $a$ & \\
\hline$\hat{b}\left[1+\left(\frac{1}{b^{r}}-\frac{1}{b} \hat{\rho}_{b}\right) \hat{\sigma}_{b b}-\frac{1}{r b} \hat{\sigma}_{b b}\left(\hat{L}_{b b b} \hat{\sigma}_{b b}+\hat{L}_{a a b} \hat{\sigma}_{a a}+\hat{L}_{\lambda \lambda b} \hat{\sigma}_{\lambda \lambda}\right)\right]^{-1}$ & $b$ & 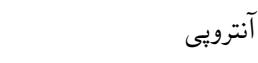 \\
\hline$\hat{\lambda}\left[\left[1+\left(\frac{1}{\hat{\lambda}^{r}}-\frac{1}{\hat{\lambda}} \hat{\rho}_{\lambda}\right) \hat{\sigma}_{\lambda \lambda}-\frac{1}{r \hat{\lambda}} \hat{\sigma}_{\lambda \lambda}\left(\hat{L}_{\lambda \lambda \lambda} \hat{\sigma}_{\lambda \lambda}+\hat{L}_{a a \lambda} \hat{\sigma}_{a a}+\hat{L}_{b b \lambda} \hat{\sigma}_{b b}\right)\right]^{-1}\right.$ & $\lambda$ & \\
\hline$\hat{a} e^{\frac{1}{r \hat{a}}\left[\left(-\frac{1}{\hat{a}}+r \hat{\rho}_{a}\right) \hat{\sigma}_{a a}+\hat{\sigma}_{a a}\left(\hat{L}_{a a a} \hat{\sigma}_{a a}+\hat{L}_{b b a} \hat{\sigma}_{b b}+\hat{L}_{\lambda \lambda a} \hat{\sigma}_{\lambda \lambda}\right)\right]}$ & $a$ & \\
\hline$\hat{b} e^{\frac{1}{r b}\left[\left(-\frac{1}{b}+r \hat{\rho}_{b}\right) \hat{\sigma}_{b b}+\hat{\sigma}_{b b}\left(\hat{L}_{b b b} \hat{\sigma}_{b b}+\hat{L}_{a a b} \hat{\sigma}_{a a}+\hat{L}_{\lambda \lambda b} \hat{\sigma}_{\lambda \lambda}\right)\right]}$ & $b$ & 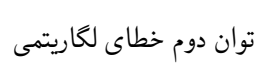 \\
\hline$\hat{\lambda} e^{\frac{1}{r \lambda}\left[\left(-\frac{1}{\lambda}+r \hat{\rho}_{\lambda}\right) \hat{\sigma}_{\lambda \lambda}+\hat{\sigma}_{\lambda \lambda}\left(\hat{L}_{\lambda \lambda \lambda} \hat{\sigma}_{\lambda \lambda}+\hat{L}_{a a \lambda} \hat{\sigma}_{a a}+\hat{L}_{b b \lambda} \hat{\sigma}_{b b}\right)\right]}$ & $\lambda$ & \\
\hline $\exp \left(\frac{1}{\hat{a}}\left[1+\frac{1}{\hat{a}}\left(\frac{1}{\hat{a}}-\hat{\rho}_{a}\right) \hat{\sigma}_{a a}-\frac{1}{r \hat{a}} \hat{\sigma}_{a a}\left(\hat{L}_{a a a} \hat{\sigma}_{a a}+\hat{L}_{b b a} \hat{\sigma}_{b b}+\hat{L}_{\lambda \lambda a} \hat{\sigma}_{\lambda \lambda}\right)\right]\right)$ & $a$ & \\
\hline $\exp \left(\frac{1}{b}\left[1+\frac{1}{b}\left(\frac{1}{b}-\hat{\rho}_{b}\right) \hat{\sigma}_{b b}-\frac{1}{r \hat{b}} \hat{\sigma}_{b b}\left(\hat{L}_{b b b} \hat{\sigma}_{b b}+\hat{L}_{a a b} \hat{\sigma}_{a a}+\hat{L}_{\lambda \lambda b} \hat{\sigma}_{\lambda \lambda}\right)\right]\right)$ & $b$ & 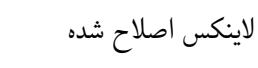 \\
\hline $\exp \left(\frac{1}{\hat{\lambda}}\left[1+\frac{1}{\hat{\lambda}}\left(\frac{1}{\hat{\lambda}}-\hat{\rho}_{\lambda}\right) \hat{\sigma}_{\lambda \lambda}-\frac{1}{r \hat{\lambda}} \hat{\sigma}_{\lambda \lambda}\left(\hat{L}_{\lambda \lambda \lambda} \hat{\sigma}_{\lambda \lambda}+\hat{L}_{a a \lambda} \hat{\sigma}_{a a}+\hat{L}_{b b \lambda} \hat{\sigma}_{b b}\right)\right]\right)$ & $\lambda$ & \\
\hline
\end{tabular}

همجنين

$L(\mathbf{x} \mid a, b, \lambda)=n b \log a+n \log b+n \log \bar{a}+n \log \lambda-\lambda \sum_{i=1}^{n} x_{i}$

$-n \log \left(1-a^{b}\right)-(b+1) \sum_{i=1}^{n} \log \left[1-e^{-\lambda x_{i}} \bar{a}\right]$ 


$$
\begin{aligned}
& \text { است. بنابراين } \\
& \hat{L}_{a a a}=\frac{\left(n b(b-1)(b-r) a^{b-r}+n b(b-1)(b+r) a^{r b-r}+r n b a^{r b-r}\right)}{\left(1-a^{b}\right)^{r}}+\frac{r n b}{a^{r}} \\
& -\frac{r n}{\bar{a}^{r}}-(b+1) \sum_{i=1}^{n} \frac{r e^{-r \lambda x_{i}}}{\left(1-e^{-\lambda x_{i}} \bar{a}\right)^{r}} \\
& \hat{L}_{b b b}=\frac{r n}{b^{r}}+\frac{n a^{b}(\log a)^{r}\left(1+a^{b}\right)}{\left(1-a^{b}\right)^{r}}, \\
& \hat{L}_{\lambda \lambda \lambda}=\frac{r n}{\lambda^{r}}-(b+1) \sum_{i=1}^{n} \frac{\bar{a} x_{i}^{r} e^{-\lambda x_{i}}\left(1+e^{-\lambda x_{i}} \bar{a}\right)}{\left(1-e^{-\lambda x_{i}} \bar{a}\right)^{r}} \text {, } \\
& \hat{L}_{b b a}=\frac{n b a^{b-1}(\log a)^{\Upsilon}(1+a)+r n a^{b-1}(\log a)(1-a)}{\left(1-a^{b}\right)^{r}}, \\
& \hat{L}_{\lambda \lambda b}=\sum_{i=1}^{n} \frac{\bar{a} x_{i}^{r} e^{-\lambda x_{i}}}{\left(1-e^{-\lambda x_{i}} \bar{a}\right)^{r}}, \\
& \hat{L}_{b b \lambda}={ }^{\circ} \text {, } \\
& \hat{L}_{a a \lambda}=-(b+1) \sum_{i=1}^{n} \frac{r x_{i} e^{-r \lambda x_{i}}}{\left(1-e^{-\lambda x_{i}} \bar{a}\right)^{r}}, \\
& \hat{L}_{\lambda \lambda a}=-(b+1) \sum_{i=1}^{n} \frac{x_{i}^{\curlyvee} e^{-\lambda x_{i}}\left(1+e^{-\lambda x_{i}} \bar{a}\right)}{\left(1-e^{-\lambda x_{i}} \bar{a}\right)^{r}}, \\
& \hat{L}_{a a b}=\frac{n(r b-1) a^{b-r}+n b(b-1) a^{b-r}(\log a)-r n(b-1) a^{r b-r}}{\left(1-a^{b}\right)^{r}} \\
& +\frac{n b(b+1) a^{\Upsilon b-r}(\log a)+n a^{r b-r}}{\left(1-a^{b}\right)^{r}}-\frac{n}{a^{\Upsilon}}+\sum_{i=1}^{n} \frac{e^{-\curlyvee \lambda x_{i}}}{\left(1-e^{-\lambda x_{i}} \bar{a}\right)^{r}} .
\end{aligned}
$$

برآورد بيزى بارامترها براى تشكيل بازه اطمينان بيزى با استفاده از نرم افزار R محاسبه و در جدول ب ارائه






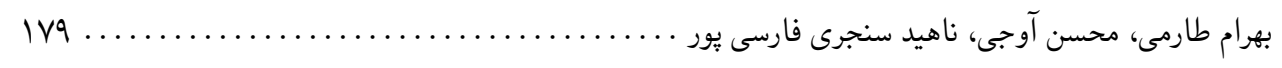

\begin{tabular}{|c|c|c|c|}
\hline \multicolumn{4}{|c|}{ جدول r. بِ رامترها براى تشكيل بازه اطمينان بيزى } \\
\hline SSE & برآورد & 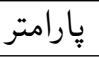 & تابع زيان \\
\hline $1 / 9 \pi$ & $r / \Psi \Lambda$ & $a$ & توان دوم خطا \\
\hline$r / 9 r$ & $r / T$ & $b$ & \\
\hline 0,001 & $1 / 01$ & $\lambda$ & \\
\hline $1 / 4 k$ & $r / Y_{0}$ & $a$ & آ آنترويى \\
\hline$r / \circ r$ & $1 / 9 V$ & $b$ & \\
\hline $0 / 001$ & $1 / 01$ & $\lambda$ & \\
\hline $1 / 40$ & $r / 41$ & $a$ & توان دوم خطاى لحاريتمى \\
\hline$\varphi / \circ \Delta$ & $1 / 91$ & $b$ & \\
\hline $0 / 001$ & $1 / 01$ & $\lambda$ & \\
\hline$\circ / I V$ & $1 / v^{c}$ & $a$ & لاينكس اصلاح شده \\
\hline $0 / 001$ & $1 / 004$ & $b$ & \\
\hline$\circ / \circ r$ & $r / V_{0}$ & $\lambda$ & \\
\hline
\end{tabular}

q خانواده توزيعهاى وايبل اصلاح شده

سه توزيع مهم از خانواده توزيعهاى وايبل اصلاح شده مورد مطالعه قرار مىگيرد. در اين خانواده از توزيعها، ه $h(x ; \lambda, \gamma)=x^{\gamma-1} \exp (\lambda x)(\gamma+\lambda x)$ و $H(x ; \lambda, \gamma)=x^{\gamma} \exp (\lambda x)$ الف- توزيع وايبل اصلاح شده كورويج: تابع توزيع، تابع جالى و و تابع مخاطره به ترتيب عبارتند از

$$
\begin{aligned}
G(x ; \alpha, \lambda, \gamma) & =1-\exp \left[-\alpha x^{\gamma} \exp (\lambda x)\right], \alpha, \lambda, \gamma>\circ, \\
g(x ; \alpha, \lambda, \gamma) & =\alpha x^{\gamma-1}(\gamma+\lambda x) \exp \left[\lambda x-\alpha x^{\gamma} \exp (\lambda x)\right], \\
r(x ; \alpha, \lambda, \gamma) & =\frac{\alpha x^{\gamma-1}(\gamma+\lambda x) \exp \left[\lambda x-\alpha x^{\gamma} \exp (\lambda x)\right]}{\exp \left[-\alpha x^{\gamma} \exp (\lambda x)\right]} \\
& =\alpha x^{\gamma-1}(\gamma+\lambda x) \exp [\lambda x] .
\end{aligned}
$$


خانواده توزيعهاى وايبل تعميميافته



(ج)

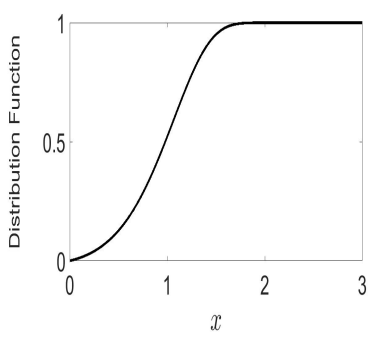

(ب)

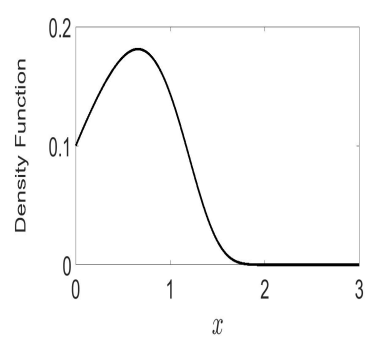

(الف)

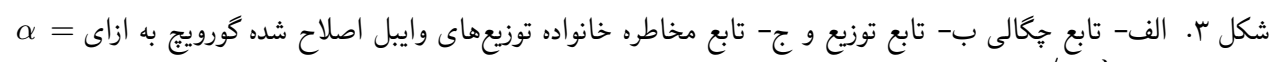
。 $/, \lambda=r, \gamma=1$

ب- توزيع وايبل اصلاح شده مارشال-اولكين: تابع توزيع، تابع جگالى و تابع مخاطره عبارتند از

$$
\begin{aligned}
G(x ; a, \alpha, \lambda, \gamma) & =\frac{1-\exp \left[-\alpha x^{\gamma} \exp (\lambda x)\right]}{1-\bar{a} \exp \left[-\alpha x^{\gamma} \exp (\lambda x)\right]}, a, \alpha, \lambda, \gamma>\circ \\
g(x ; a, \alpha, \lambda, \gamma) & =\frac{a \alpha x^{\gamma-1}(\gamma+\lambda x) \exp \left[\lambda x-\alpha x^{\gamma} \exp (\lambda x)\right]}{\left(1-\bar{a} \exp \left[-\alpha x^{\gamma} \exp (\lambda x)\right]\right)^{\gamma}} \\
r(x ; a, \alpha, \lambda, \gamma) & =\frac{\alpha x^{\gamma-1} \exp (\lambda x)(\gamma+\lambda x)}{1-\bar{a} \exp \left[-\alpha x^{\gamma} \exp (\lambda x)\right]}
\end{aligned}
$$

ج- توزيع وايبل اصلاح شده مارشال-اولكين-ناداراجه: تابع جگالى، تابع توزيع و تابع نرخ مخاطره



(ج)

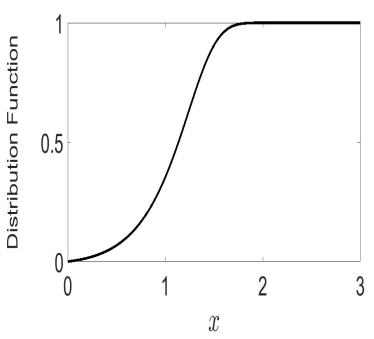

$(ب)$



(الف)

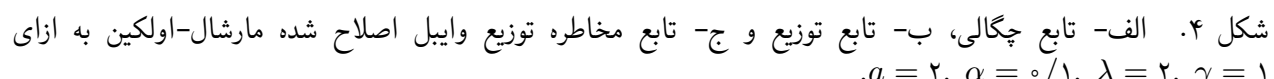




\section{اين توزيع به ترتيب عبارتند از}

$$
\begin{aligned}
g(x ; a, b, \alpha, \lambda, \gamma) & =\frac{a^{b} b \bar{a} \alpha x^{\gamma-1}(\gamma+\lambda x) \exp \left[\lambda x-\alpha x^{\gamma} \exp (\lambda x)\right]}{\left(1-a^{b}\right)\left[1-\bar{a} \exp \left[-\alpha x^{\gamma} \exp (\lambda x)\right]\right]^{b+1}}, \lambda, \gamma>\circ, \\
G(x ; a, b, \alpha, \lambda, \gamma) & =\frac{1}{1-a^{b}}-\frac{a^{b}}{1-a^{b}}\left[1-\bar{a} \exp \left[-\alpha x^{\gamma} \exp (\lambda x)\right]\right]^{-b}, \\
r(x ; a, b, \alpha, \lambda, \gamma) & =\frac{b \bar{a} \alpha x^{\gamma-1} \exp (\lambda x)(\gamma+\lambda x) \exp \left[-\alpha x^{\gamma} \exp (\lambda x)\right]}{\left[1-\bar{a} \exp \left[-\alpha x^{\gamma} \exp (\lambda x)\right]\right]\left\{1-\left[1-\bar{a} \exp \left[-\alpha x^{\gamma} \exp (\lambda x)\right]\right]^{b}\right\}}
\end{aligned}
$$

اين توزيع به نماد TPMOMW $(a, b, \alpha, \lambda, \gamma$ نمايش داده مىشود. r امين گشتاور اين مدل به صورت

$$
E\left(X^{r}\right)=\sum_{j=。}^{\infty} w_{j} \mu_{r}(j)
$$

$$
w_{j}= \begin{cases}\eta_{j}, & \circ<a<1 \\ \nu_{j}, & a>1\end{cases}
$$

$$
\begin{aligned}
\mu_{r}(j) & =\int_{0}^{\infty} x^{r} f(x ;(j+1) \alpha, \lambda, \gamma) d x \\
& =\int_{0}^{\infty} x^{r}(j+1) \alpha x^{\gamma-1} \exp (\lambda x)(\gamma+\lambda x) \exp \left[-\alpha(j+1) x^{\gamma} \exp (\lambda x)\right] d x \\
\eta_{j} & =\frac{(b+1)_{j}}{(j+1) !} a^{b} b \bar{a}^{j+1}, \quad \nu_{j}=\frac{(-1)^{j}}{(j+1) !} \frac{b \bar{a}}{\left(1-a^{b}\right)^{a}} \sum_{k=j}^{\infty} \frac{(b+1)_{k}}{(k-j) !}\left(1-\frac{1}{a}\right)^{k}
\end{aligned}
$$

كارراسكو و همكارانش (Y०ᄉ) يك نمايش از (j)

$$
\mu_{r}(j)=\sum_{i_{1}, \ldots, i_{r}=1}^{\infty} \frac{A_{i_{1}, \ldots, i_{r}} p\left(\frac{s_{r}}{\gamma}+1\right)}{[(j+1) \alpha]^{\frac{s_{r}}{\gamma}}}
$$

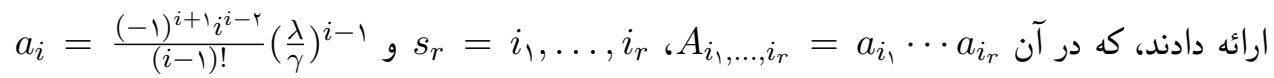
هستند. حال فرض كنيد 
خانواده توزيعهاى وايبل تعميميافته

$$
\text { باشند. لعاريتم تابع درستنمايى براى بردار پارامترهاى (a, }
$$

$$
\begin{aligned}
\ell(\theta)= & n b \log a+n \log b+n \log \bar{a}+n \log \alpha-n \log \left(1-a^{b}\right) \\
& +(\gamma-1) \sum_{i=1}^{n} \log x_{i}+\lambda \sum_{i=1}^{n} x_{i}+\sum_{i=1}^{n} \log \left(\gamma+\lambda x_{i}\right) \\
& -\alpha \sum_{i=1}^{n} x_{i}^{\gamma} \exp \left(\lambda x_{i}\right)-b \log \left[1-\bar{a} \exp \left[-\alpha x_{i}^{\gamma} \exp \left(\lambda x_{i}\right)\right]\right]
\end{aligned}
$$

است. نمودارهاى تابع جگالى و تابع مخاطره توزيع وايبل اصلاح شده مارشال-اولكين-ناداراجه به ازاى دو حالت

$\operatorname{TPMOMW}(r, \circ / \Delta, r, r, 1 / \Delta)$

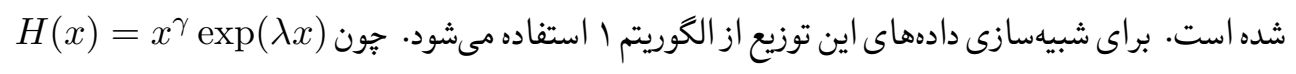

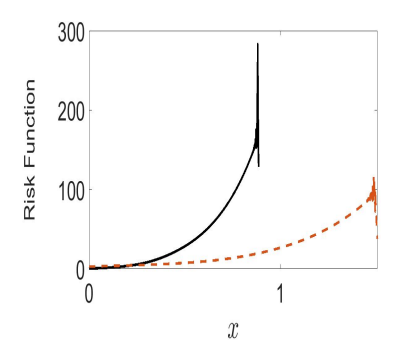

(ج)

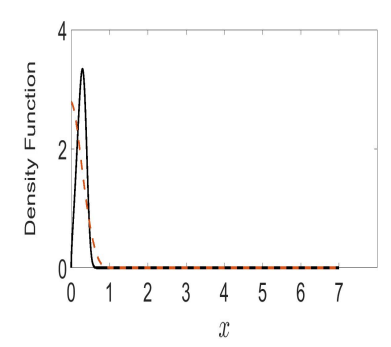

(ب)

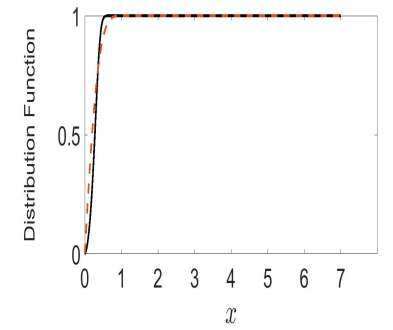

(الف)

شكل ه. الف- تابع توزيع، ب- تابع جاكالى و ج- تابع مخاطره خانواده توزيعهاى وايبل اصلاح شده مارشال-اولكين-ناداراجه

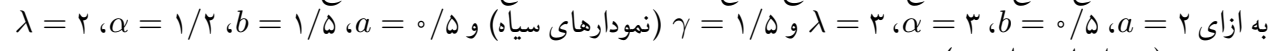
و



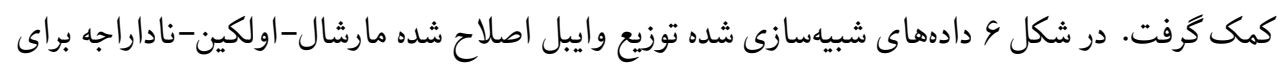
TPMOMW(r, 。/ $/ \Delta, r, r, 1 / \Delta)$ 
بهرام طارمى، محسن آوجى، ناهيد سنجرى فارسى يور .

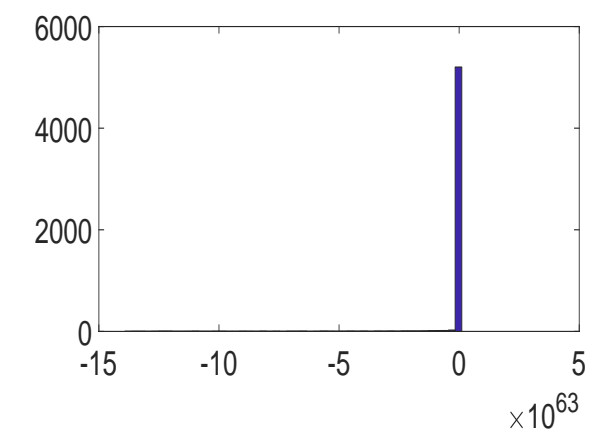

(ب)

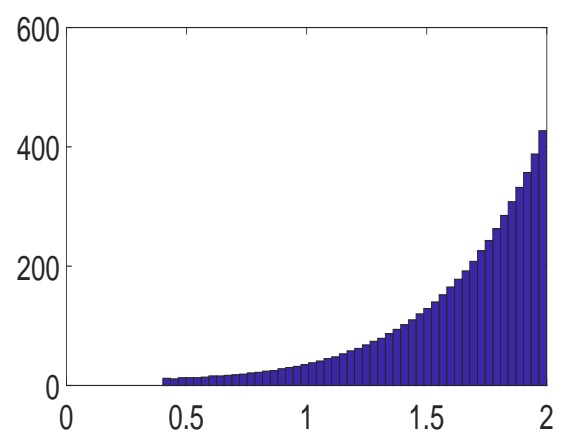

(الف)

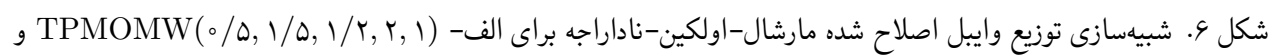
TPMOMW(

\section{خانواده توزيعهاى گمبرتز V V}

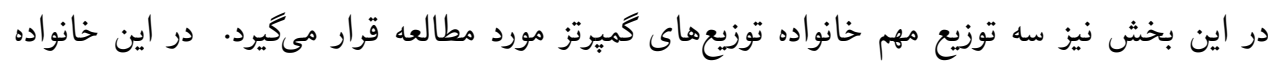

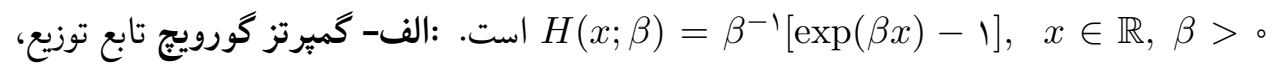
تابع حكالى و تابع مخاطره اين توزيع، بترتيب عبارتند از

$$
\begin{aligned}
& G(x ; \alpha, \beta)=1-\exp \left[-\alpha \beta^{-1}[\exp (\beta x)-1]\right], \\
& g(x ; \alpha, \beta)=\alpha \exp (\beta x) \exp \left[-\alpha \beta^{-1}[\exp (\beta x)-1]\right], \\
& r(x ; \alpha, \beta)=\alpha \exp (\beta x) .
\end{aligned}
$$

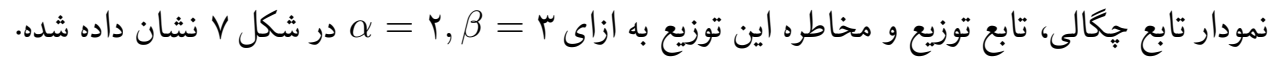

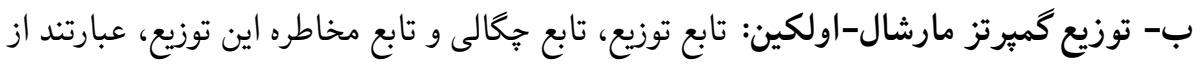






(ج)

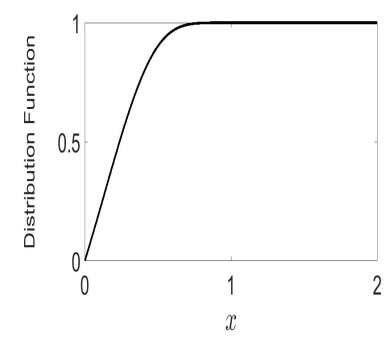

(ب)



(الف)

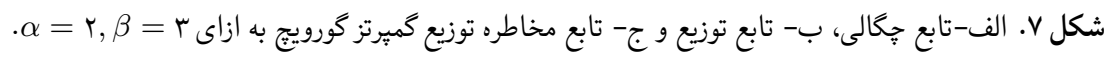

$$
\begin{aligned}
& G(x ; a, \alpha, \beta)=\frac{1-\exp \left[-\frac{\alpha}{\beta}[\exp (\beta x)-1]\right]}{1-\bar{a} \exp \left[-\frac{\alpha}{\beta}[\exp (\beta x)-1]\right]}, \\
& g(x ; a, \alpha, \beta)=\frac{a \alpha \exp \left[\beta x-\frac{\alpha}{\beta}[\exp (\beta x)-1]\right]}{1-\bar{a} \exp \left[-\frac{\alpha}{\beta}[\exp (\beta x)-1]\right]^{r}}, \\
& r(x ; a, \alpha, \beta)=\frac{\alpha \exp (\beta x)}{1-\bar{a} \exp \left[-\frac{\alpha}{\beta}[\exp (\beta x)-1]\right]} .
\end{aligned}
$$

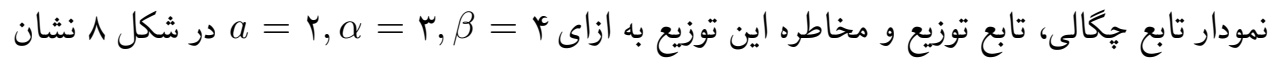

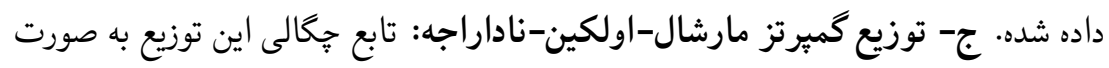

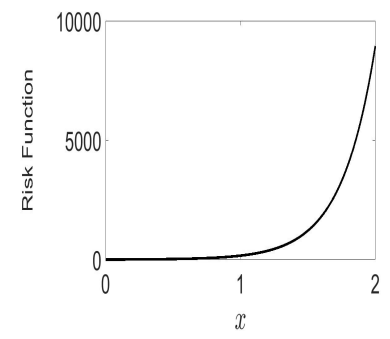

(ج)



(ب)



(الف)

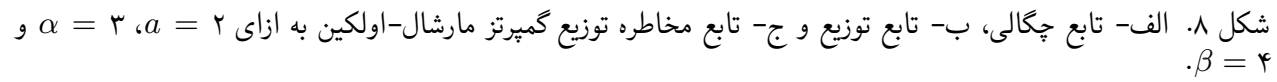



$g(x ; a, b, \alpha, \beta)=\frac{a^{b} b \bar{a} \alpha \exp (\beta x) \exp \left[-\alpha \beta^{-1}[\exp (\beta x)-1]\right]}{\left(1-a^{b}\right)\left[1-\bar{a} \exp \left[\alpha \beta^{-1}[\exp (\beta x)-1]\right]\right]^{b+1}}, \quad \beta \in \mathbb{R} \backslash\{\circ\}, x \in \mathbb{R}^{+}$,

است. توزيع گمبرتز مارشال-اولكين-ناداراجه را با نماد TPMOGO نمايش داده مىشود. تابع توزيع و

$$
\text { تابع نرخ مخاطره اين مدل به ترتيب عبارتند از مارسات }
$$

$$
\begin{aligned}
G(x ; a, b, \alpha, \beta) & =\frac{1}{1-a^{b}}-\frac{a^{b}}{1-a^{b}}[1-\bar{a} R]^{-b}, \\
r(x ; a, b, \alpha, \beta) & =\frac{b \alpha \bar{a} \exp (\beta x) R}{(1-\bar{a} R])\left(1-[1-\bar{a} R]^{b}\right)}
\end{aligned}
$$

كه در آنها [ 1 - - أن

$$
\text { از اين توزيع باشند. لكاريتم تابع درستنمايى (a, }
$$

$$
\begin{aligned}
\ell(\theta)= & n b \log a+\log b+\log \bar{a}+\log \alpha+\beta \sum_{i=1}^{n} x_{i}-\alpha \beta^{-1} \sum_{i=1}^{n} \exp \left(\beta x_{i}\right) \\
& +\alpha \beta^{-1} n-\log \left(1-a^{b}\right)-\sum_{i=1}^{n} \log \left[1-\bar{a} \exp \left[-\alpha \beta^{-1}\left[\exp \left(\beta x_{i}\right)-1\right]\right]\right] .
\end{aligned}
$$

است. نمودار تابع جگالى، تابع توزيع و مخاطره به ازاى

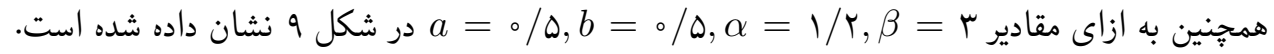
نمودار مقادير شبيهسازى شده از اين توزيع با استفاده از الكوريتم ا و [1 - - (x)

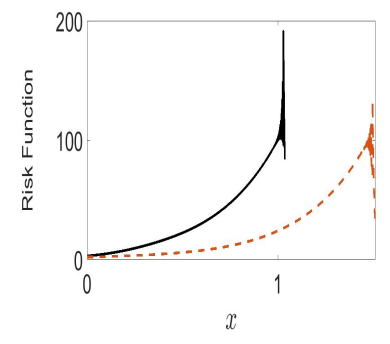

(ج)

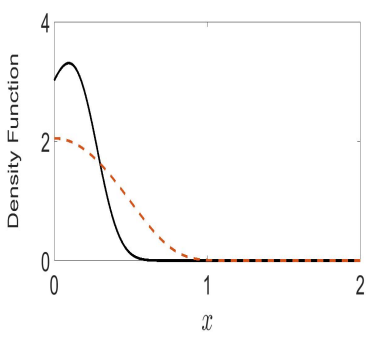

(ب)



(الف)

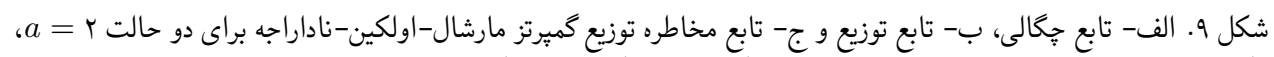

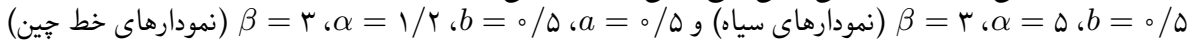


براى دو حالت مذكور در شكل ه ا نشان داده شده است. $H^{-1}(x)=\beta^{-1} \log (\beta x+1)$
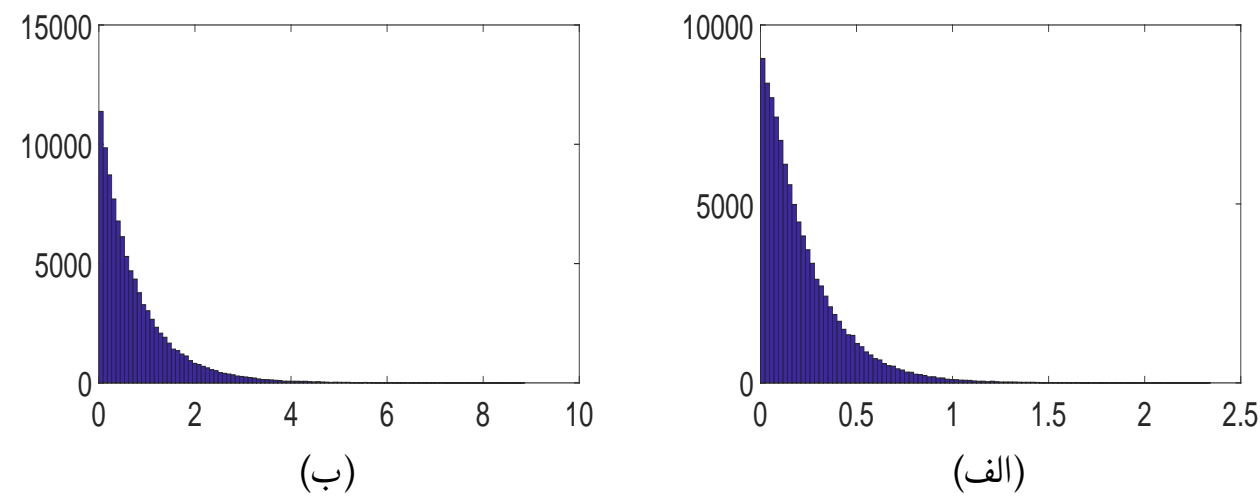

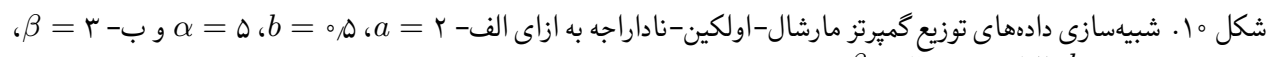
$\beta=r, \alpha=1, r ،=\circ, b ، a=\circ / \Delta$

\section{^ تحليل دادهاى بيمه آتش سوزى}

در اين بخش توزيع هاى معرفى شده به دادهاى بيمه آتش سوزى دانمارك برازش داده شده و يارامترهاى آنها با

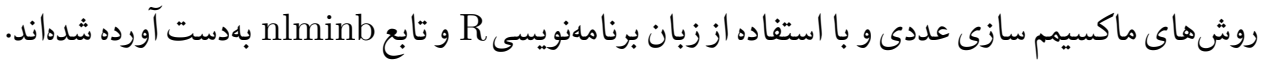

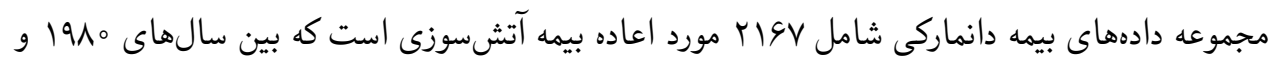

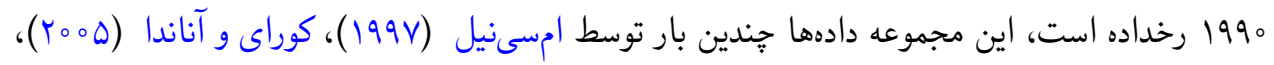

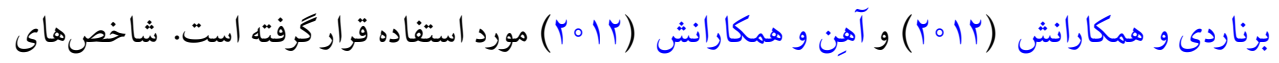

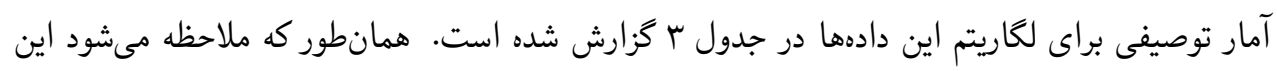

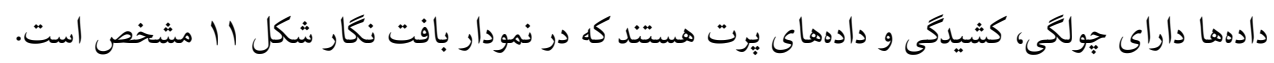

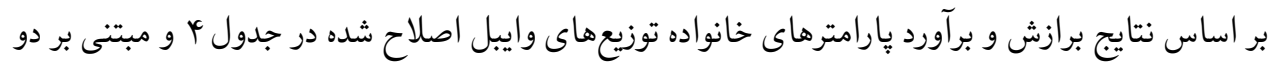

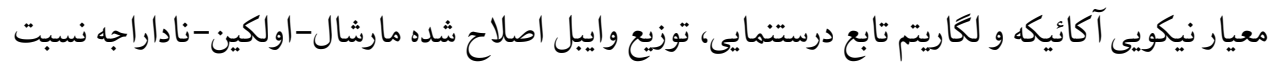

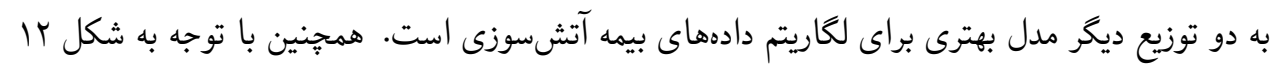
توزيع وايبل اصلاح شده مارشال-اولكين مدل مناسبترى دون نسبت به به توزيع وايبل اصلاح شده كورويج است. با دقت به اين نمودارها مشخص مىشود كه توزيع وايبل اصلاح شده مارشال-اولكين-ناداراجه خيلى بهتر

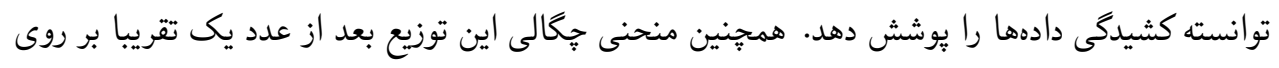


بهرام طارمى، محسن آوجى، ناهيد سنجرى فارسى يور

\begin{tabular}{|c|c|}
\hline مقدار & شاخص \\
\hline $0 / \sqrt{ } 9$ & ميانگين \\
\hline ONT & انحرف استاندارد \\
\hline ING & جولى \\
\hline 4,11 & كشيدگى \\
\hline $0,0 \circ$ & مينيمم \\
\hline $0, Y \Lambda$ & جارك اول \\
\hline$\circ / \Delta 9$ & ميانه \\
\hline 1,09 & جارك سوم \\
\hline$\Delta / \Delta V$ & ماكسيمم \\
\hline
\end{tabular}

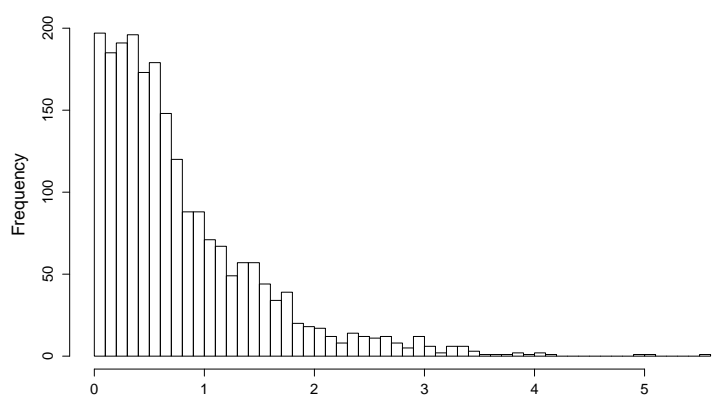

شكل 11 ال. بافتنكار لكاريتم دادهاى بيمه آتشسوزى دانمارى.

مستطيلها نشسته است و اين موضوع نشان مىدهد كه اين توزيع مدل مناسبى براى اين دادهها است. با

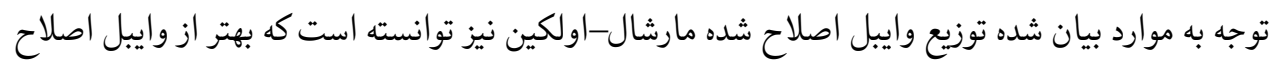

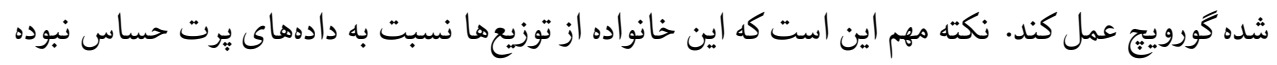

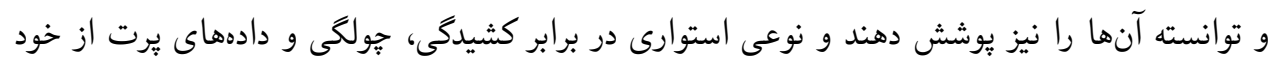
نشان دادهاند.

جدول ه نيز براورد پارامترهاى خانواده توزيعهاى گميرتز را نشان مىدهد. با توجه به اين جدول و و






\begin{tabular}{|c|c|c|c|c|c|c|c|}
\hline AIC & $-\log l i k$ & $b$ & $a$ & $\gamma$ & $\lambda$ & $\alpha$ & توزيع \\
\hline TYNIANI & TIVVYAT & - & - & 1,NYOV & $0,9 \mathrm{TH}$ & oAMKY & وايبل اصلاح شده كورويج \\
\hline$T \forall \times Y Y, 90$ & ITVISAT & - & $0, A r 90$ & 0,9409 & $1, / T O 1$ & $09 \Delta M K$ & وايبل اصلاح شده مارشال-اولكين \\
\hline KYTOQ/M & $1 \% 9909$ & D,MCY & 0,4949 & $1, \pi V A K$ & ०N9TD & $\circ 9 \vee 90$ & وايبل اصلاح شده مارشال-اولكين-ناداراجه \\
\hline
\end{tabular}

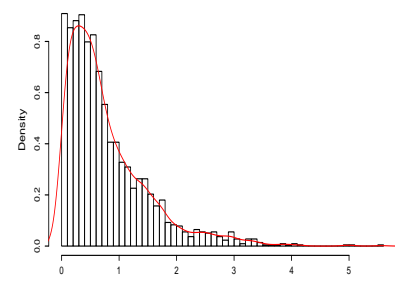

(ج)

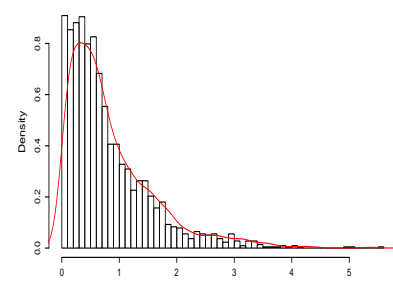

(ب)

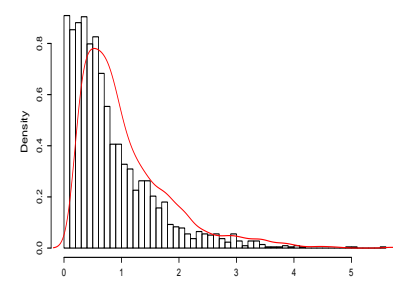

(الف)

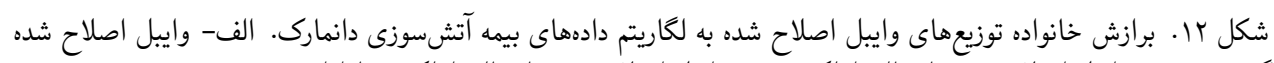
كورويج، ب- وايبل اصلاح شده مارشال-اولكين، ج- وايبل اصلاح شده مارشال-اولكين-ناداراجه.

از دو توزيع ديكر اين خانواده است و با توجه به نمودارهاى شكل ه مشخص مىشود همانند توزيع وايبل

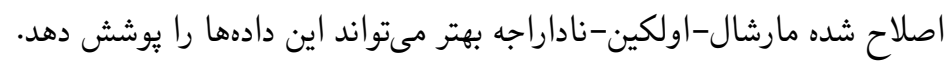

\begin{tabular}{|c|c|c|c|c|c|c|}
\hline AIC & $-\log l i k$ & $b$ & $a$ & $\beta$ & $\alpha$ & توزنع \\
\hline$r \Delta \Delta r \Delta, r q$ & ITVGON & - & - & I,TEYT & $1,99 \mathrm{VI}$ & كميرتزكورويجج \\
\hline$r \Delta r q \Lambda, 09$ & ITVYG,OH & - & $\circ \Delta T \Delta Y$ & ITSOY & $\circ 9 \circ V_{0}$ & كميرتزمارشال-اولكين \\
\hline$r F V H \Lambda, H Y$ & IrrGo/IV & roVIT & 0 TrgVt & - 9 TLS & -NDHE & گميرتزمارشال-اولكين-ناداراجه \\
\hline
\end{tabular}

\section{9}

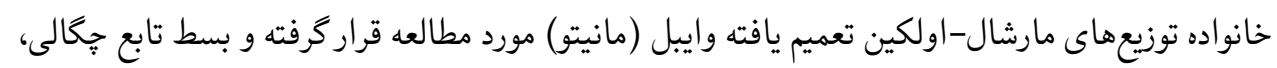



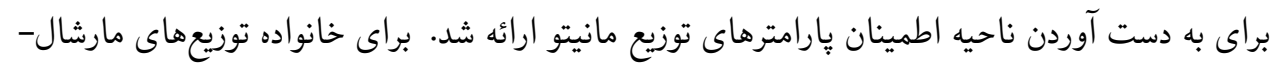




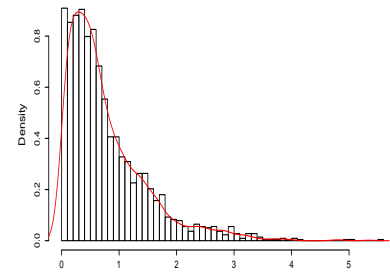

(ج)



(ب)

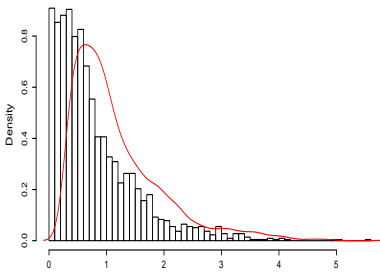

(الف)

شكل rا. برازش خانواده توزيعهاى كميرتز به لكاريتم دادههاى بيمه آتشسوزى دانمارك. الف- كميرتز كورويج، ب- كميرتز مارشال-اولكين، ج-كميرتز مارشال-اولكين-ناداراجه.

اولكين-ناداراجه نمايى (مانن) توابع بقاء، جآلى و مخاطره معرفى و نجوه شبيهسازى از اين توزيع بيان

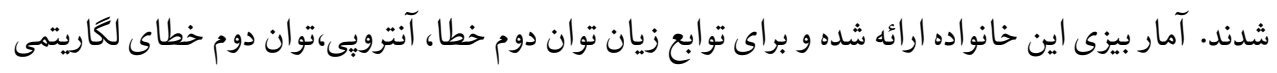

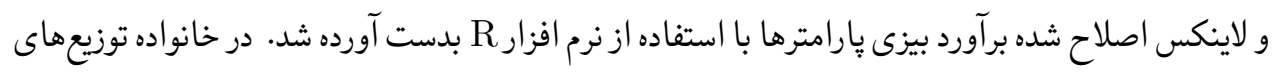

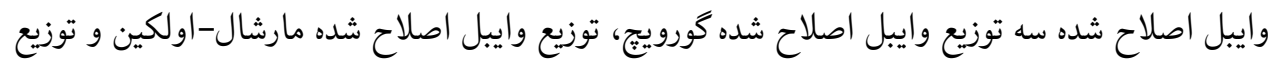







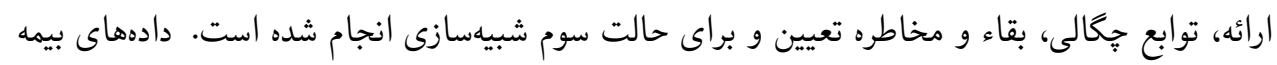



\section{تقدير و تشكر}

از هيئت تحريريه، داوران و ويراستار محترم مجله كه با توصيههاى ارزشمند خود سبب ارتقا و ارائه بهتر اين مقاله شدند كمال تشكر را داريم.

\section{مراجع}

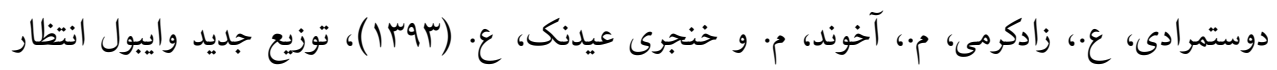

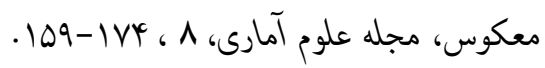




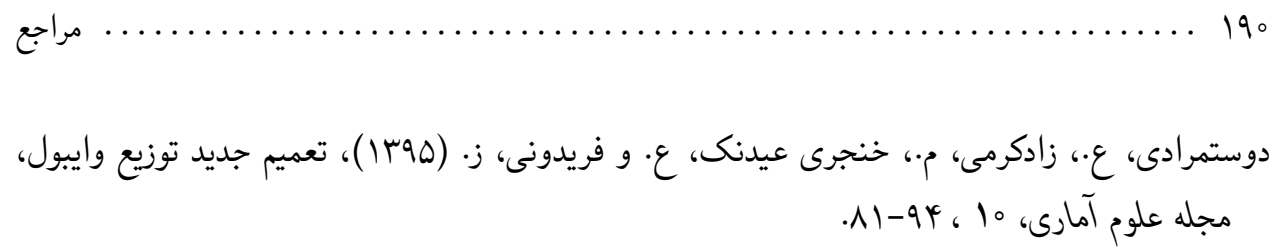

Ahn, S., Kim, J. H.T., and Ramaswami, V. (2012), A New Class of Models for Heavy Tailed Distributions in Finance and Insurance Risk, Insurance: Mathematics and Economics, 51, 43-52.

Bernardi, M., Maruotti, A., and Petrella, L. (2012), Skew Mixture Models for Loss Distributions: A Bayesian Approach, Insurance: Mathematics and Economics, 51, 617-623.

Carrasco, J. M. F., Orteya, E. M. M., and Cordeir, G. M. (2008), A Generalized Modified Weibull Distribution for Lifetime Modeling, Computational Statistics and Data Analysis, 53, 450-462.

Cooray, K., and Ananda, M. M. A. (2005), Modeling Actuarial Data with a Composite Lognormal-Pareto Model, Scandinavian Actuarial Journal, 2005, 321-334.

Cover, T. M., and Thomas, J. A. (1991), Elements of Information Theory, John Wiley and Sons, New York.

Cox, D. R., and Hinkley, D. V. (1974), Theoretical Statistics, Chapman and Hall, London.

Gurvich, M., DiBenedetto, A., and Ranade, S. (1997), A New Statistical Distribution for Characterizing the Random Strength of Brittle Materials, Journal of Materials Science, 32, 2559-2564.

Jayakumar, K., and Thomas, M. (2008), On a Generalization to MarshalOlkin Scheme and Its Application to Burr Type XII Distribution, Statistical Papers, 49, 421-439. 
Lindley, D. V. (1980), Approximate Bayes Method, Trabajos de Estadistica, 31, 223-237.

Marshall, A. W., and Olkin, I. (1997), A New Method for Adding a Parameter to a Family of Distributions with Application to the Exponential and Weibull Families, Biometrika, 84, 641-652.

McNeil, A. J. (1997), Estimating the Tails of Loss Severity Distributions Using Extreme Value Theory, ASTIN Bulletin: The Journal of the IAA, 27 (1), 117-137.

Nadarajah, S., Jayakumar, K., and Ristic, M. M. (2012), A New Family of Lifetime Models, Journal of Statistical Computation and Simulation, 83, 1389-1404.

Nascimento, A. D. C., Cintra, R. J., and Frery, A. C. (2010), Hypothesis Testing in Speckled Data with Stochastic Distances, IEEE Transactions on Geoscience and Remote Sensing, 48, 373-385.

Santos-Neto, M., Bourguignon, M., Zea, L. M., and Nascimento, A. D. C. (2014), The Marshall-Olkin Extended Weibull Family of Distributions, Joural of Statistical Distributions and Applications, 1, 1-24.

Singh, S. k., Singh, U., and Yadav, A. S. (2014), Bayesian Estimation of Marshall-Olkin Extended Exponential Paoameters Under Various Approximation Techniques, Hacettepe Journal of Mathematics and Statistics, 43, 341-354. 
Journal of Statistical Sciences, Spring and Summer, 2021

Vol. 15, No. 1, pp 165-191

DOI: $10.29252 /$ jss.15.1.165

\title{
Distributions Family of Extended Weibull Combined with Negative Binomial Distribution Truncated at Zero
}

\author{
Tarami $^{1}$, B., Avaji ${ }^{2}$, M., Sanjari Farsipour ${ }^{3}$, N. \\ ${ }^{1}$ Department of Statistics, Shiraz university, Shiraz, Iran. \\ ${ }^{2}$ Department of Applied Mathematics, Tabriz University, Tabriz, Iran. \\ ${ }^{3}$ Department of Statistics, Alzahra University, Tehran, Iran.
}

\begin{abstract}
In this paper, using extended Weibull Marshall-Olkin-Nadarajah family of distributions, the exponential, modified Weibull, and Gompertz distributions are obtained, and density, survival, and hazard functions are simulated. Next, an algorithm is presented for the simulation of these distributions. For exponential case, Bayesian statistics under squared error, entropy Linex, squared error loss functions and modified Linex are calculated. Finally, the presented distributions are fitted to a real data set.
\end{abstract}

Keywords: Marshal-Olkin distribution, Generalized Weibull distribution, Bayesian statistics, Survival function, Hazard function.

Mathematics Subject Classification (2010): 62F15, 62C10, $60 \mathrm{E} 05$. 